\title{
High-Dose-Rate Vaginal Cuff Brachytherapy
}

National Cancer Institute

\section{Source}

National Cancer Institute. High-Dose-Rate Vaginal Cuff Brachytherapy. NCI Thesaurus.

Code C158399.

High dose rate radiation delivered by a radiation source contained in an intra-vaginal

cylinder to target neoplasms of the vaginal cuff. 